\title{
Do Different Textual Enhancement Formats Have Differential Effects on the Intake of English Subjunctive Mood?
}

\author{
Aliakbar Khomeijani Farahani \\ Department of English, University of Tehran, Iran \\ Email: farahani@ut.ac.ir \\ Mehdi Sarkhosh \\ Department of English, University of Tehran, Iran \\ Email: mdsarkhosh@gmail.com
}

\begin{abstract}
This research was inspired by Simard's (2009) study on the differential effects of textual enhancement on the noticing and intake of English plural markers. This study investigated the differential effects of different textual enhancement formats (typographical cues) on the intake of English subjunctive mood among EFL learners in an Iranian context. The participants were 114 female upper-intermediate learners of English in an English institute. A reading text was prepared for five experimental and one control group. For each group the target structure was enhanced differently (underline, bold, italic, back ground, and choice) in the text and for the control group the passage was intact. The last two experimental formats, i.e., back ground and choice, were created by the present researchers to examine their effectiveness on the intake of the target structure. ANOVA analysis and Scheffe post hoc test were applied to analyze the data. Final results revealed that, in comparison with other formats, underline textual enhancement format was more effective in inducing the intake of target structure. However, back ground and choice did not prove to be effective textual enhancement formats.
\end{abstract}

Index Terms - textual enhancement, noticing, intake, subjunctive mood

\section{INTRODUCTION}

The teaching of grammar has been the locus of hot disputes among language teaching gurus for a long age, the question is not whether to teach grammar or not but is how to teach it from among a wide range of pedagogical options open to language practitioners (Ellis, 1997). The argument in SLA has been in favor of integrating grammar instruction into communicative language teaching (Pica, 2000; Savignon, 1991). One way to this end is through input enhancement which leads to increased attention on the part of learner and therefore increased noticing (Sharwood Smith, 1981).

Noticing has received considerable attention from applied linguistics researchers in the past two decades (e.g., Schmidt \& Frota, 1986; Sharwood Smith, 1981, 1991, 1993). Qi and Lapkin define noticing "as the awareness of stimulus via short-term memory." They refer to stimulus as "anything that rouses one's attention." (Qi and Lapkin, 2001, p.279). There is a consensus among applied linguists that attention to form is necessary for the acquisition of form (e.g., Doughty, 1991; Fotos, 1994, 1998; Nassaji, 1999; Lightbown and Spada, 1990). Making a structural feature salient by enhancing that feature induces this attention on the part of learners. According to Sharwood Smith (1991, 1993) attention is paid to form when the form is made more salient which brings about noticing and subsequent intake of the enhanced form.

One way to bring about noticing is through Textual Enhancement (TE) whose main function is to draw learners' attention to linguistic forms by modifying the physical appearance of target structures. The use of typographical cues such as bold, italic, capital, underlining, changing the size of the font of letters and highlighting is the most typical way of enhancing the saliency of certain linguistic features in written texts (Simard, 2009). TE is making a particular linguistic feature more salient in the text in order to make the reader notice this feature. TE of a particular item in the text makes the learner pay more attention to that item which, in turn, is said to trigger noticing. Intake of a particular linguistic feature is the result of learner's paying attention to that feature. These features, when made salient or enhanced are more likely to be paid attention to (Schmidt, 1994a,b, 1995, 2001). A number of empirical studies endorse the view that when higher amount of attention is paid to form, more learning takes place (Leow, 1997b; Robinson, 1996; Rosa and O'Neill, 1999; Schmidt and Frota, 1986).

Concerning previous studies focusing on TE, it should be noted that the issue of the TE impact on intake and acquisition has remained an area of controversy among researchers. Some studies reject the positive effect of TE on intake, comprehension or acquisition (e.g., Alanen, 1995; Leow, 1997a; Leow, 2001) and some are in favor of the influential role of TE in noticing, intake or the acquisition of linguistic features (e.g., Jourdenaise, Stauffer, Boyson \& 
Doughty, 1995; Shook, 1994). This study, however, is an attempt to shed more light on the influence of TE on learners' acquisition of linguistic features by introducing two new TE formats which, to the best knowledge of the authors, are unprecedented in TE literature. One is back grounding in which the whole reading text is bolded and the target features are back grounded or dimmed as in the sentence taken from the reading text used in this study: "... I suggested that she go through the usual channels and get a bank loan...". The second format was to introduce both correct and incorrect forms in the reading text while the incorrect forms were identified by an asterisk and come after the correct forms. The latter is less implicit in nature compared to other TE formats, but it is not utterly explicit in that there is no explanation as to how the target structure works. The following sentence is taken from the reading text used in this study: “... I suggested that she (go/goes*) through the usual channels and get a bank loan...".

One limitation of this study is that since these two TE formats are novel, there is no relevant literature to be investigated by the researchers. These two TE formats, are derived from general beliefs about human nature (explained below) and do not have theoretical background in the literature. However, there are no strong claims made regarding their effectiveness. They were systematically and experimentally tested and the final results revealed their ineffectiveness. The underlying reasons for selecting the two new formats by the researchers of the study are as follows: The authors chose background TE format based on the common belief in human nature in that whatever is less available is more coveted and this inclination might tempt learners to pay more attention to dimmed parts of a text. In other words, the researchers presumed that the sense of curiosity might cause more noticing to occur for less noticeable parts of the text. The choice TE format was selected based on the belief that moderation is the best policy, that is, we believe TE is very implicit and metalinguistic explanations are utterly explicit. Choice TE format seems to be the half way between and is a compromise between implicit TE and explicit metalinguistic explanations.

Simard's (2009) work on the differential effects of TE on the intake of English plural markers has inspired this work and the present researchers have drawn on his design and methodology in some parts of this study. However, this study is by no means a replication of Simard (2009). The present researchers have conducted a similar research in the Iranian context on a different group of participants with different features from those of Simard in terms of their proficiency level. The target structure is also different. The number and types of typographical cues are also different. In addition, this study investigates two novel TE formats which have not been investigated before neither by Simard nor by any other researcher.

\section{LITERATURE REVIEW}

TE studies have mostly been controlled experiments with a pre-test, post-test design and post test usually following immediately (Han, Park \& Combs, 2008). Studies mostly targeted a particular linguistic feature such as relative clauses (Izumi, 2002), Spanish preterit versus imperfect forms (Jourdenais et al., 1995), or English plural markers (Simard, 2009). What is obvious concerning TE is that the field is still full of controversies and contradictions. The following is a review of some of the major studies.

\section{A. Studies Concurring Little or No Impact of TE on Noticing, Intake, or Acquisition}

Alanen (1995) studied the effects of TE (italics) and explicit rule presentation on learners' processing of semi artificial Finnish locative suffixes and consonant gradation. Sentence completion and grammaticality judgment task were utilized in order to measure the subjects' knowledge of target forms. Think aloud protocols were employed to measure noticing and rule awareness. The final results revealed that although the performance of subjects exposed to TE was significantly different from that of the control group, compared to explicit rule presentation group, their performance was lower. Leow (1997a) inspected the effects of TE (underline and bold) and passage length on 84 college level learners of Spanish. There were four conditions (long, enhanced; short, enhanced; long, unenhanced; short, unenhanced) to which subjects were exposed. To measure their intake, the subjects were given a short answer comprehension task and a multiple-choice recognition task. The findings demonstrated no effect of TE on either comprehension or intake. A couple of years later, Leow (2001) investigated the effect of TE (underline and bold) on the learning of imperative forms among 38 Spanish learners. On-line think aloud protocols, a recognition task and a written production task were used; however, there was no effect of TE on intake or comprehension. Overstreet (1998) examined the effect of TE (bold, underline, enlarged letters and different font) and content familiarity on learners' intake of preterit and imperfect tenses in Spanish and on their comprehension of passage content. Not only did he find no effect of TE on subjects' intake of target features, but also he found a negative effect of TE on comprehension. Jourdenais (1998), likewise, came to the same conclusion. She studied the effect of TE (underline, bold, shadow, and different font) on 124 learners' intake of Spanish preterite and imperfect tense. These subjects had to read three chapters of narration in one of the four conditions (enhanced preterit, enhanced imperfect, unenhanced preterit, and unenhanced imperfect). By using a production task in which the subjects were asked to write an essay, data were collected but the results were disappointing regarding the effect of TE. The effect of TE (enlargement, different combinations of bold, italics, and underline) on the ability to use third person singular possessive determiners was investigated by White (1998). The participants' ability to use possessive determiners was measured by using oral picture description, passage correction task and a multiple-choice task. The final findings divulged that TE did not result in the correct use of the target features while increasing the frequency of the use of these features. Izumi (2000; cited in Leow, 2001) studied the effect of TE 
(bold, shadow, different fonts, and sizes) on subjects' ability to evaluate relativization in the passages they were given. To assess the effect of TE, he used note-taking, sentence combination task with picture cued essay and post exposure questionnaire, but he found no learning gains from pretest to posttest. Leow, Nuevo and Tsai (2003) investigated the role of TE (underline, bold, and enlarged fonts) and language features (present, perfect, and subjunctive) on comprehension and intake of 72 adults who were learning Spanish. Learners' noticing and intake were measured by think-aloud protocols, a multiple-choice recognition task and a multiple-choice comprehension task. Their results did not reveal any effect of TE on noticing, comprehension or intake. Wong (2000, 2003) examined the effect of TE (bold, underline, italics, and enlarged letters) and input simplification on the acquisition of past participle agreement among 81 English learners of French. Error identification and correction task was applied to measure learning and a free recall task to measure comprehension; nonetheless, no effect of TE was found on the acquisition of the intended structure.

\section{B. Studies Concurring the Positive Effect of TE on Noticing, Intake, or Acquisition}

In an study on the effect of TE (bold and capital) on 125 first and second year English speaking Spanish learners' intake of present perfect and relative pronouns, Shook (1994) employed two off-line tasks (multiple-choice recognition and fill in the blank production) to measure intake. He had three groups (one control and two experimental). Two written production tasks (one for the present perfect and one for the relative pronouns) and two written recognition tasks (one for the present perfect and one for the relative pronouns) were used. The experimental groups which were exposed to TE performed significantly better than the control group but the difference between the scores of the two experimental groups was not significant. Jourdenais et al. (1995) investigated the effect of TE (underline, bold, shadow, and different font) of preterit and imperfect tense in Spanish on the noticing of 10 English first year learners of Spanish. Think aloud protocols and a written task were used as measurement instruments. Overall, the results revealed that subjects exposed to TE significantly performed better than the control group by reporting more episodes containing the targeted linguistic feature. Izumi (2003) explored the impact of TE (different font, different size of fonts, shadow, and bold) on the noticing and learning of relative clauses by adult English learners. Noticing was assessed by utilizing the notes subjects took during exposure to the experimental treatment. A grammaticality judgment, sentence combination task and an interpretation task were also employed to measure the subjects' knowledge of relative clauses. No learning was reported in the post test although subjects demonstrated noticing of the target forms. Lee (2007) examined the effect of TE on the acquisition and comprehension of meaning among 259 Korean English learners. To measure intake, a correction task and to measure comprehension, a free recall task were applied respectively. He found that TE lead to the acquisition of the target forms but had a negative effect on comprehension. Simard (2009) studied the influence of TE by enhancing the same text by eight different conditions (italic, underline, capital, bold, color, 3-cues, 5-cues, and control). She examined the differential effect of TE on French English learners' acquisition of English plural markers. He concluded that capital and three-cue group performed better than the other groups.

A prominent characteristic of TE studies is that the results regarding the effectiveness of TE vary so greatly (Izumi, 2003; Lee, 2007; Lee \& Huang, 2008). As Han et al. (2008) put it, methodological idiosyncrasies are the norm of this body of research. They maintain that seven major issues appear to be limiting the generalizability of the findings and holding up further progress in understanding the efficacy of TE for learning: (1) noticing and/or acquisition, (2) TE and comprehension, (3) simultaneous or sequential processing, (4) TE and the nature of the enhanced form, (5) TE and prior knowledge, (6) TE and input flood, and (7) TE and overuse. The contradictory results obtained in TE studies can also be traced back to the differences in the methodological choices made by the authors (Simard, 2009). Simard refers to the fact that linguistic features selected, languages observed, measurement instruments used and constructs examined in these studies vary greatly.

The review of the literature on TE reveals that, to the researchers' best knowledge, no study to date has been conducted on the effect of TE on Persian learners' acquisition of English subjunctive mood. As part of previous literature on TE (part 2.2.) demonstrates, TE is conducive to learning grammatical structures of a language. Regarding English grammatical structures, subjunctive mood is an area of difficulty for Persian learners and it's a complex linguistic feature for them. This difficulty has been observed by the authors and their colleagues via the experience of teaching at different English institutes. Hence, this study is an attempt to investigate the influence of TE on the acquisition of English subjunctive mood by upper-intermediate Persian learners of English. In addition, there are two novel and unprecedented TE formats introduced- back ground and choice (as explained in introduction)- in order to investigate the effect of TE on intake through these new approaches and compare them with mainstream TE formats (e.g., bold, italic, capital, different color, different font, etc). To fulfill the aim of the study, the following research question was raised:

- Do different TE formats (typographical cues) have differential effects on Persian learners' intake of English subjunctive mood?

\section{METHOD}

\section{A. Participants}

114 female Iranian EFL learners who were learning English in an English institute in Orumie, a city in north west of Iran, participated in the study. They were all female, their age ranged between 14 and $41(\mathrm{M}=17.39, \mathrm{SD}=4.85)$ and 
their level of education varied from high school to M.A. All subjects were Turkish speakers who were proficient in both Turkish and Persian. In English, they were at the upper-intermediate level of proficiency, that is, they had studied English for 3 years in that institute: three and half hours of instruction per week (two days a week; each day, one hour and forty five minutes). The Participants had taken the placement test when enrolling in this institute. In addition, in the end of each term they were given an achievement test in order to let the qualified ones pass onto the next level. Therefore, they were almost at the same level of English proficiency. However, in order to make sure that they are equal, at least, in terms of their knowledge of English subjunctive mood, a pre test was given and the results revealed that there were no between group differences at pre test.

\section{B. Instruments}

\section{Background Questionnaire}

In order to obtain the necessary demographical information about subjects such as, age, educational level, years of studying English, etc, a background questionnaire was employed.

\section{Reading Text}

A reading text of 326 words at upper-intermediate level was chosen. For the control group, there was no intervention and the text was neutral; however, for each of the five experimental groups the subjunctive structures inside the text were enhanced differently, respectively, italics, bold, underline, back grounding and choice. In order to make sure that the text was of appropriate level of difficulty, it was piloted on an upper-intermediate group of learners who had the same features as the target groups. In so doing, the text was administered to a class of upper-intermediate learners with 23 students in the same institute. In addition to asking learners orally about the difficulty of the text, ten comprehension questions followed the text. Learners' oral reports confirmed the appropriateness of the text for their level. Also, the comprehension questions were answered 78 percent correctly by learners which was suggestive of the fact that the text was almost well comprehended by the pilot group. In piloting the text, the researchers also examined the reliability of the text by applying KR-21 formula which turned out to be 0.76 .

\section{Multiple-Choice Recognition Tests}

Two parallel versions of a multiple-choice recognition test were constructed (A \& B), one for pre-test and one for post-test, as this method is most commonly used to investigate the effect of TE on intake (Leow, 1997; Overstreet, 1998). Furthermore, by employing multiple-choice test, the researchers prevent subjects form utilizing avoidance strategies and direct their performance toward the intended structures. Each version of the test consisted of eighteen multiple choice questions, twelve on subjunctive and six fillers. The equivalence of two tests was confirmed during a pilot study in which the twelve subjunctive questions of pretest and posttest were put together into one test of 24 questions on English subjunctive mood. Odd numbers were assigned pretest questions and even numbers posttest questions to ensure the best mixture of pretest and posttest questions. The 24 multiple choice questions were given to an advanced class of EFL learners at the same institute who had already learned subjunctive structures according to the institute's syllabus and their teachers' reports to the researchers. The mean of the class was 84 which indicated that the subjects had an ample knowledge of subjunctive. The two parts of the test that is, odd numbers (pretest questions) and even numbers (posttest questions) were scored separately. In order to investigate if the two versions were parallel the correlation coefficient between two parts (odd numbers and even numbers) was computed which turned out to be 0.84 .

\section{Target Structure}

The English subjunctive mood was the target structure on which the effect of differential types of TE was investigated. English subjunctive mood is used in American English in sentences such as " It is important that she study her lessons." or "it is necessary that she do her homework." This structure, as mentioned before, is a complex one and is a source of difficulty for Iranian learners. This difficulty was observed by the researchers and also their colleagues teaching throughout the country.

\section{Procedure}

The study was carried out in Iran Language Institute, one of the oldest and most well known language teaching centers in Iran between November $12^{\text {th }}$ and December $1^{\text {st }}, 2010$. Before conducting the research, the researchers organized a meeting with the teachers who were supposed to administer the study in their classes to inform them about the exact administration process they were supposed to follow. To make sure that the subjects had no knowledge of English subjunctive mood, the researchers analyzed the syllabi they had covered till then and also asked teachers whether they had taught subjunctive mood to their students already or whether their students had any knowledge about the topic. Then, the data were collected in two sessions. The first session, the teachers administered the demographical information questionnaire and the pretest in 20 minutes. The pretest was one of the two versions of the multiple choice recognition test (A or $\mathrm{B}$ ) and was administered to ensure that subjects had no prior knowledge of subjunctive and to verify if all groups were equal and comparable at the pretest. The scores of subjects on the pre-test revealed that they had almost no knowledge of English subjunctive mood and there was no significant difference between the six groups that participated in the study. Next session, the reading passage was administered. The text was enhanced differently for different groups. The subjects read the passages in 20 minutes and the posttest immediately followed which took 10 minutes. The posttest was the version of the multiple-choice recognition test that subjects had not completed during the 
pretest. To score the test, each correct answer was given three points. Why three points was chosen to be given to each correct answer was decided only on an arbitrary basis. As stated earlier, there were eighteen questions for pre-test and eighteen for post-test. The questions were parallel out of which six were fillers and twelve were on subjunctive mood in both tests. Only the twelve subjunctive questions were corrected by the researchers and as was mentioned each correct answer was given three points. Therefore, the subjects' scores ranged from zero to thirty-six in each test.

\section{E. Data Analysis}

To ensure the normality of the distribution, descriptive statistics was run. To see the difference of the mean scores among the six groups on pretest, posttest and the difference between pre-test and post-test, a one-way ANOVA was conducted to the data. To identify the precise location of the differences, a Scheffe's test was applied.

\section{RESULTS}

The descriptive statistics for the six groups at the pre-test are displayed in Table 1.

TABLE-1:

DESCRIPTIVE STATISTICS OF THE SIX GROUPS AT PRE-TEST

\begin{tabular}{|c|c|c|c|c|c|c|c|c|}
\hline & \multirow[b]{2}{*}{$\mathbf{N}$} & \multirow[b]{2}{*}{ Mean } & \multirow{2}{*}{\multicolumn{2}{|c|}{ Std. Deviation Std. Error }} & \multicolumn{2}{|c|}{ 95\% Confidence Interval for Mean } & \multirow[b]{2}{*}{ Minimum } & \multirow[b]{2}{*}{ Maximum } \\
\hline & & & & & Lower Bound & Upper Bound & & \\
\hline Control & 24 & 8.25 & 5.252 & 1.072 & 6.03 & 10.47 & 0 & 18 \\
\hline Background & 16 & 9.38 & 5.784 & 1.446 & 6.29 & 12.46 & 0 & 21 \\
\hline Bold & 16 & 8.06 & 5.335 & 1.334 & 5.22 & 10.91 & 0 & 21 \\
\hline Underline & 21 & 8.71 & 6.141 & 1.340 & 5.92 & 11.51 & 0 & 24 \\
\hline Choice & 14 & 9.86 & 4.912 & 1.313 & 7.02 & 12.69 & 0 & 21 \\
\hline Italic & 23 & 8.35 & 4.951 & 1.032 & 6.21 & 10.49 & 0 & 21 \\
\hline Total & 114 & 8.68 & 5.330 & .499 & 7.70 & 9.67 & 0 & 24 \\
\hline
\end{tabular}

To compare the mean scores of the six groups at the pre-test, a one-way ANOVA was run. The $F$-observed value and p-value were .274 and .926 , respectively. This amount of $F$-value at 5 and 108 degrees of freedom was lower than the critical value of $F$ (i.e., 4.40) and p-value was higher than the significance level of .05 (see Table 2):

TABLE-2:

ONE-WAY ANOVA ON THE SIX GROUPS AT PRE-TEST

\begin{tabular}{llllll}
\hline & Sum of Squares & df & Mean Square & F & Sig. \\
\hline Between Groups & 40.227 & 5 & 8.045 & .274 & .926 \\
Within Groups & 3170.405 & 108 & 29.356 & & \\
Total & 3210.632 & 113 & & & \\
\hline
\end{tabular}

Therefore, it can be concluded that there was no significant difference between the mean scores of the six groups at pre-test $(\mathrm{F}(5,108)=.274, \mathrm{p}>.05)$.

The result of the Levene's test of homogeneity of variance revealed that the six groups enjoyed homogenous variance; that is, there was not any marked difference between the variance of the six groups. Hence, the results of the one-way ANOVA were reliable $(\mathrm{F}(5,108)=.419, \mathrm{p}>.05)$ (see Table 2$)$ :

The descriptive statistics for the six groups at the post-test are illustrated in Table 3.

TABLE-3:

DESCRIPTIVE STATISTICS OF THE SIX GROUPS AT POST-TEST

\begin{tabular}{|c|c|c|c|c|c|c|c|c|}
\hline & \multirow[b]{2}{*}{$\mathbf{N}$} & \multirow[b]{2}{*}{ Mean } & \multirow{2}{*}{\multicolumn{2}{|c|}{ Std. Deviation Std. Error }} & \multicolumn{2}{|c|}{$95 \%$ Confidence Interval for Mean } & \multirow[b]{2}{*}{ Minimum } & \multirow[b]{2}{*}{ Maximum } \\
\hline & & & & & Lower Bound & Upper Bound & & \\
\hline Control & 24 & 12.63 & 9.486 & 1.936 & 8.62 & 16.63 & 0 & 36 \\
\hline Background & 16 & 9.00 & 3.950 & .987 & 6.90 & 11.10 & 3 & 15 \\
\hline Bold & 16 & 17.81 & 8.448 & 2.112 & 13.31 & 22.31 & 6 & 33 \\
\hline Underline & 21 & 22.43 & 7.972 & 1.740 & 18.80 & 26.06 & 9 & 33 \\
\hline Choice & 14 & 11.86 & 8.393 & 2.243 & 7.01 & 16.70 & 0 & 33 \\
\hline Italic & 23 & 15.52 & 7.971 & 1.662 & 12.07 & 18.97 & 3 & 36 \\
\hline Total & 114 & 15.14 & 8.973 & .840 & 13.48 & 16.81 & 0 & 36 \\
\hline
\end{tabular}

To compare the mean scores of the six groups at the post-test, a one-way ANOVA was conducted. The F-observed value and p-value were 6.64 and 0.000 respectively. This amount of F-value at 5 and 108 degrees of freedom was higher than the critical value of $F$, and p-value was lower than the significance level of .05 $(\mathrm{F}(5,108)=6.640, \mathrm{p}<.05)$ (see Table 4): 
TABLE-4:

ONE-WAY ANOVA ON THE SIX GROUPS AT POST-TEST

\begin{tabular}{llllll}
\hline & Sum of Squares & df & Mean Square & F & Sig. \\
\hline Between Groups & 2139.096 & 5 & 427.819 & 6.640 & .000 \\
Within Groups & 6958.659 & 108 & 64.432 & & \\
Total & 9097.754 & 113 & & & \\
\hline
\end{tabular}

Thus, it can be concluded that there is a significant difference between the mean scores of the six groups on post-test. The effect size, calculated via eta squared, was found to be 0.23 . This indicates the degree of association between the dependent (post-test scores) and independent (the different types of TE) variable, which is a large size (Dornyei, 2007).

The result of the Leven's test of homogeneity of variance demonstrated that the six groups had homogenous variance $(\mathrm{F}(5,108)=1.881, \mathrm{p}>.05)$; accordingly, the results of the one-way ANOVA were reliable, that is, there was not any marked difference between the variance of the six groups.

ANOVA analysis revealed that there is a difference somewhere among the means, but the precise location of differences is not clear. To locate the exact place of differences, a post hoc comparison of the means was performed. In so doing, a Scheffe's test was applied. The results of the post-hoc Scheffe's test indicated that, at the level of 0.05 , there was significant difference between the Underline group and the three groups of Control, Background and Choice (see Table 5).

TABLE-5:

SCEFFE'S TEST FOR THE COMPARISON OF POST TEST MEANS OF THE DIFFERENT GROUPS

\begin{tabular}{|c|c|c|c|c|c|c|}
\hline \multirow[b]{2}{*}{ (I) GROUP } & \multirow[b]{2}{*}{ (J) GROUP } & \multirow{2}{*}{$\begin{array}{l}\text { Mean Difference } \\
\text { (I-J) }\end{array}$} & \multirow[b]{2}{*}{ Std. Error } & \multirow[b]{2}{*}{ Sig. } & \multicolumn{2}{|c|}{$\mathbf{9 5 \%}$ Confidence Interval } \\
\hline & & & & & Lower Bound & Upper Bound \\
\hline \multirow[t]{5}{*}{ Control } & Background & 3.63 & 2.591 & .854 & -5.16 & 12.41 \\
\hline & Bold & -5.19 & 2.591 & .551 & -13.97 & 3.59 \\
\hline & Underline & $-9.80(*)$ & 2.399 & .008 & -17.93 & -1.67 \\
\hline & Choice & .77 & 2.699 & 1.000 & -8.38 & 9.92 \\
\hline & Italic & -2.90 & 2.342 & .908 & -10.84 & 5.04 \\
\hline \multirow{5}{*}{ Background } & Control & -3.63 & 2.591 & .854 & -12.41 & 5.16 \\
\hline & Bold & -8.81 & 2.838 & .095 & -18.43 & .81 \\
\hline & Underline & $-13.43(*)$ & 2.664 & .000 & -22.46 & -4.40 \\
\hline & Choice & -2.86 & 2.938 & .966 & -12.82 & 7.10 \\
\hline & Italic & -6.52 & 2.613 & .293 & -15.38 & 2.34 \\
\hline \multirow[t]{5}{*}{ Bold } & Control & 5.19 & 2.591 & .551 & -3.59 & 13.97 \\
\hline & Background & 8.81 & 2.838 & .095 & -.81 & 18.43 \\
\hline & Underline & -4.62 & 2.664 & .700 & -13.65 & 4.41 \\
\hline & Choice & 5.96 & 2.938 & .537 & -4.00 & 15.91 \\
\hline & Italic & 2.29 & 2.613 & .979 & -6.57 & 11.15 \\
\hline \multirow[t]{5}{*}{ Underline } & Control & $9.80(*)$ & 2.399 & .008 & 1.67 & 17.93 \\
\hline & Background & $13.43(*)$ & 2.664 & .000 & 4.40 & 22.46 \\
\hline & Bold & 4.62 & 2.664 & .700 & -4.41 & 13.65 \\
\hline & Choice & $10.57(*)$ & 2.770 & .017 & 1.18 & 19.96 \\
\hline & Italic & 6.91 & 2.423 & .159 & -1.31 & 15.12 \\
\hline \multirow[t]{5}{*}{ Choice } & Control & -.77 & 2.699 & 1.000 & -9.92 & 8.38 \\
\hline & Background & 2.86 & 2.938 & .966 & -7.10 & 12.82 \\
\hline & Bold & -5.96 & 2.938 & .537 & -15.91 & 4.00 \\
\hline & Underline & $-10.57(*)$ & 2.770 & .017 & -19.96 & -1.18 \\
\hline & Italic & -3.66 & 2.721 & .873 & -12.89 & 5.56 \\
\hline \multirow[t]{5}{*}{ Italic } & Control & 2.90 & 2.342 & .908 & -5.04 & 10.84 \\
\hline & Background & 6.52 & 2.613 & .293 & -2.34 & 15.38 \\
\hline & Bold & -2.29 & 2.613 & .979 & -11.15 & 6.57 \\
\hline & Underline & -6.91 & 2.423 & .159 & -15.12 & 1.31 \\
\hline & Choice & 3.66 & 2.721 & .873 & -5.56 & 12.89 \\
\hline
\end{tabular}

To investigate the impact of the different types of TE on the intake of the intended grammatical structure more precisely, the difference of scores at pre-test and post-test was calculated and the related statistical analyses were conducted to them. The descriptive statistics for the difference of scores at pre-test and post-test for the six groups are illustrated in Table 6. 
TABLE-6:

DESCRIPTIVE STATISTICS FOR THE DIFFERENCE OF SCORES AT PRE-TEST AND POST-TEST

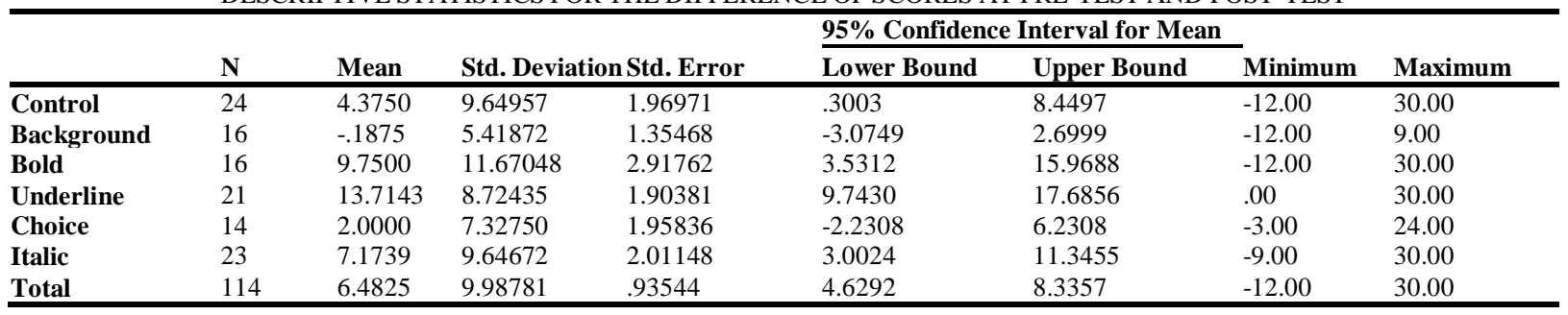

To compare the mean scores of the difference at pre-test and post-test for the six groups, a one-way ANOVA was conducted. The F-observed value was 5.780. This amount of F-value at 5 and 108 degrees of freedom was higher that the critical value of F, i.e., 4.40 (see Table 7).

TABLE-7:

ONE-WAY ANOVA ON THE DIFFERENCE OF SCORES AT PRE-TEST AND POST-TEST

\begin{tabular}{llllll}
\hline & Sum of Squares & df & Mean Square & F & Sig. \\
\hline Between Groups & 2379.812 & 5 & 475.962 & 5.780 & .000 \\
Within Groups & 8892.653 & 108 & 82.339 & & \\
Total & 11272.465 & 113 & & & \\
\hline
\end{tabular}

Thus, there is a significant difference between the mean scores of the difference at pre-test and post-test for the six groups $(\mathrm{F}(5,108)=5.780, \mathrm{p}<.05)$. The effect size, calculated via eta squared, was found to be 0.21 . This magnitude points out the degree of connection between the dependent (the difference of scores at pre-test and post-test) and independent (the different types of TE) variable, which is a large size (Dornyei, 2007). The result of the Levene's test of homogeneity of variance revealed that the six groups enjoyed homogenous variance; consequently, the results of the one-way ANOVA were reliable. The $F$-value of 1.842 at 5 and 108 degrees of freedom was lower than the critical value of 4.40. Thus, the underlying assumption of one-way ANOVA was met, that is, there was not any marked difference between the variance of the three groups $(\mathrm{F}(5,108)=1.842$, $\mathrm{p}>.05)$.

To locate the exact place of differences, a Scheffe's test was utilized. The results indicated that, at the level of 0.05 , there was significant difference between the Underline group and the three groups of Control, Background and Choice (see Table 8).

TABLE-8:

SCEFFE'S TEST FOR THE COMPARISON OF DIFFERENCE OF MEANS AT PRETEST AND POSTTEST FOR THE SIX GROUPS

\begin{tabular}{|c|c|c|c|c|c|c|}
\hline \multirow[b]{2}{*}{ (I) GROUP } & \multirow[b]{2}{*}{ (J) GROUP } & \multirow{2}{*}{$\begin{array}{l}\text { Mean } \\
\text { Difference(I-J) }\end{array}$} & \multirow[b]{2}{*}{ Std. Error } & \multirow[b]{2}{*}{ Sig. } & \multicolumn{2}{|c|}{ 95\% Confidence Interval } \\
\hline & & & & & Lower Bound & Upper Bound \\
\hline \multirow[t]{5}{*}{ Control } & Background & 4.5625 & 2.92865 & .787 & -5.3657 & 14.4907 \\
\hline & Bold & -5.3750 & 2.92865 & 644 & -15.3032 & 4.5532 \\
\hline & Underline & $-9.3393(*)$ & 2.71141 & .044 & -18.5310 & -.1476 \\
\hline & Choice & 2.3750 & 3.05159 & .987 & -7.9699 & 12.7199 \\
\hline & Italic & -2.7989 & 2.64779 & .952 & -11.7749 & 6.1771 \\
\hline \multirow[t]{5}{*}{ Background } & Control & -4.5625 & 2.92865 & .787 & -14.4907 & 5.3657 \\
\hline & Bold & -9.9375 & 3.20818 & .097 & -20.8133 & .9383 \\
\hline & Underline & $-13.9018(*)$ & 3.01117 & .001 & -24.1097 & -3.6939 \\
\hline & Choice & -2.1875 & 3.32078 & .994 & -13.4450 & 9.0700 \\
\hline & Italic & -7.3614 & 2.95401 & .295 & -17.3755 & 2.6527 \\
\hline \multirow[t]{5}{*}{ Bold } & Control & 5.3750 & 2.92865 & .644 & -4.5532 & 15.3032 \\
\hline & Background & 9.9375 & 3.20818 & .097 & -.9383 & 20.8133 \\
\hline & Underline & -3.9643 & 3.01117 & .883 & -14.1722 & 6.2436 \\
\hline & Choice & 7.7500 & 3.32078 & .371 & -3.5075 & 19.0075 \\
\hline & Italic & 2.5761 & 2.95401 & .979 & -7.4380 & 12.5902 \\
\hline \multirow[t]{5}{*}{ Underline } & Control & 9.3393(*) & 2.71141 & .044 & .1476 & 18.5310 \\
\hline & Background & $13.9018(*)$ & 3.01117 & .001 & 3.6939 & 24.1097 \\
\hline & Bold & 3.9643 & 3.01117 & .883 & -6.2436 & 14.1722 \\
\hline & Choice & $11.7143(*)$ & 3.13086 & .020 & 1.1006 & 22.3279 \\
\hline & Italic & 6.5404 & 2.73878 & .343 & -2.7441 & 15.8248 \\
\hline \multirow[t]{5}{*}{ Choice } & Control & -2.3750 & 3.05159 & .987 & -12.7199 & 7.9699 \\
\hline & Background & 2.1875 & 3.32078 & .994 & -9.0700 & 13.4450 \\
\hline & Bold & -7.7500 & 3.32078 & .371 & -19.0075 & 3.5075 \\
\hline & Underline & $-11.7143(*)$ & 3.13086 & .020 & -22.3279 & -1.1006 \\
\hline & Italic & -5.1739 & 3.07593 & .726 & -15.6013 & 5.2535 \\
\hline \multirow[t]{5}{*}{ Italic } & Control & 2.7989 & 2.64779 & .952 & -6.1771 & 11.7749 \\
\hline & Background & 7.3614 & 2.95401 & .295 & -2.6527 & 17.3755 \\
\hline & Bold & -2.5761 & 2.95401 & .979 & -12.5902 & 7.4380 \\
\hline & Underline & -6.5404 & 2.73878 & .343 & -15.8248 & 2.7441 \\
\hline & Choice & 5.1739 & 3.07593 & .726 & -5.2535 & 15.6013 \\
\hline
\end{tabular}

* The mean difference is significant at the .05 level. 


\section{DISCUSSION}

The results of the present study corroborate many studies conducted in the field (e.g., Jourdenaise et al., 1995; Shook, 1994) in that the use of different TE types has differential effect on the noticing of linguistic features in the text. For example, the use of capital letters is said to make learners remember the information they are presented in their first language (Shebilske \& Rotondo, 1981). In Mark's (1966, cited in Simard, 2009) study, as well, the type and combination of the typographical cues used made subjects react differently to the instructions. Foster and Coles (1977) also found that the type of typographical cue can induce different reactions from the subjects to the task they are asked to perform.

This study investigated the differential effects of different TE formats on Persian learners' intake of English subjunctive mood. As was perspicuous at pretest, there was no significant difference between the six groups but at posttest a significant between group difference emerged. The underline group outperformed other groups at posttest which signifies that underline TE format helped learners notice the target structure better than other formats. In other words, TE format, that is, the type of typographical cue proved to be differentially effective in inducing the noticing and intake of the target form. This result confirms the findings of Simard's (2009) study. In a similar vein, according to the findings of her study, she reached to the conclusion that different types of TE format had differential effect on French English learners' intake of English plural markers.

Jourdenaise et al. (1995) and White (1996), as well, found underline an effective TE format in inducing the noticing and intake of target features. Contemplating the possible reasons for finding the underline group to outperform other groups with a large mean difference, the researchers came across the following main reasons: when we underline a structural feature, we make it more salient than when it is bolded or italicized since in underlining we add something to the text, that is, the line drawn under the target feature. In simpler words, underlining is an additive TE format in that the subject sees something extra in addition to the target structure and this is what, we conjecture, induces the noticing of what the researcher means to be noticed. However, a bolded or italicized feature might not attract as much attention as underlining triggers since the two former approaches to TE do not add anything extra to trigger the subjects' curiosity and induce their noticing of the target structure.

The second reason might be lurking behind subjects' learning strategies. To put it in a nutshell, Iranian learners at almost all levels and fields have one learning strategy in common and that is the fact that the majority underline the salient parts of their textbooks when studying for a test or during the term. This is not a claim but a learning strategy which is very popular in Iran among learners. To corroborate this assumption, at least among the participants of the present study, the researchers required the teachers to ask participants which one of the six formats they usually apply for their own learning when they are trying to emphasize the salient and important parts of the textbooks they are studying. According to the teachers' oral reports to the researchers, the majority of participants reported underlining and highlighting. This part was done orally because it was not the focus of this study and was only conducted as a probable hint for future research. Therefore, the reason why underlining proved so effective in inducing the noticing and subsequent acquisition of the target structure seems to be the participants' learning strategies and a sense of affinity they felt with this TE format.

Considering bold group, the between group mean difference at posttest was the second highest after the underline group, but it was not significant. This finding concurs the results of Leow (1997) and Overstreet (1998)'s studies. Likewise, these researchers found no effect of bold TE format on intake.

A surprising result obtained was the post test mean for the background group (9.00) which was lower than the pretest mean (9.38); however, this mean difference is infinitesimal and insignificant. What this might entail is that background TE format seems to be detrimental to the intake of target structures. Literature on the two new TE types used in this study is almost non-existent and this is why researchers find it difficult to anchor the findings, at this part, to the previous research. The reason as to why back grounding proved not only ineffective but also detrimental might reside in the fact that this format might have had negative psychological effect on subjects since bolding of the whole text and dimming the target structures makes the text difficult to read since the whole text is bolded and reading a bold text is far more difficult than reading a text in small case letters. In addition, we are all used to reading texts written in small case letters and not the other way round and this might be the reason to account for the bad performance of background group at posttest.

What present researchers find far from easy to account for is why choice TE format, like back ground, did not prove to be effective in bringing about the noticing or intake of the target structure. The only wild guess we can offer is that by introducing both correct and incorrect forms to subjects they began wondering why a particular form was incorrect and the other one was correct. Since there were no metalinguistic explanations about how the target structure workd or why the asterisk marked structure was incorrect, the subjects were baffled and this fact per se, we surmise, contributed to their malperformance. Choice TE format allowed neither implicit acquisition nor deductive conscious learning since it was half way between implicit TE and explicit metalinguistic explanation. The other reason contributing to the subjects' malperformance might be the fact that in other TE formats they read the text and rarely stop at enhanced points to figure out what is going on. However, at choice TE format they had to pause at each enhanced structure and ponder what the story was that one form was asterisk marked as incorrect and the other one was not asterisk marked and was considered correct. This is what, we reckon, has also contributed to the subjects' bad performance. 
The two novel TE formats, namely, back ground and choice did not turn out to be effective in inducing the noticing or intake of target features. Having run Scheffe's post hoc test, the researchers detected the differences to be lying among control and underline, back ground and underline, and choice and underline. The other two formats - italics and bold - were not significantly different from underline and this indicates that these two TE formats are relatively more effective than the researchers' devised ones which turned out to be significantly different from underline. Bold and italics triggered the noticing of target forms more than choice and background although this was not significant. This fact shows that the traditional TE formats, that is, underline, bold and italics are still more effective compared to background and choice.

\section{CONCLUSION}

The findings of this study indicate that a) Enhancing a particular feature in a text is effective in triggering the noticing of that feature and its subsequent intake, this finding corroborates some previous research regarding the positive effects of TE on intake such as Jourdenaise et al., (1995), Shook (1994) and Lee (2007); b) different TE formats have differential effects on noticing and intake, as Simard (2009) puts it, there is an inherent saliency potential in each TE format and they impact attention differently; c) the present researchers' devised TE formats did not impact noticing and intake of the target form, while the two most commonly used and popular TE formats, that is, underline and bold (though not significant) proved more effective.

An important implication of this study is for researchers to pay more attention to the learning strategies of learners. As was observed in this study, students reported that they usually used underlining or highlighting in order to emphasize important points in their textbooks, and as the results indicated, underline TE format was more effective than other ones in inducing noticing and intake. Highlight TE format where the target structure was highlighted was not used in this study. Had it been used, it might have been effective in triggering the intake of target structure. Bold TE format looks like highlighting more than other formats used in this study and as the result section revealed bold group's performance at the posttest was the second highest after the underline group, although not significant. Repercussions can be that a particular TE format per se might not be always effective for all learners, but what makes a particular TE format effective is how this format interacts with a learner's learning style and how well it resembles the way the learner herself emphasizes important points in a text. This will entail implications for further research which should unravel the nature of the relationship between learners' learning strategies and different TE formats. The present authors do not discuss this relationship since it is not the central focus of this study and will wait for further research to shed more light on it.

The implications for material developers are numerous. They have a wide range of TE formats at their disposal while the research results regarding their effectiveness and even differential effects vary greatly (Izumi, 2003; Lee, 2007; Lee \& Huang, 2008). According to the findings of this study, underline can be an appropriate TE format for material developers. They can apply underline TE format to the structures they want to introduce in reading texts to help learners notice them. A further implication of this study for material developers is that they should apply country or nation specific TE formats in reading texts since it seems that learners' learning strategies interact with the type of TE format they notice best; however, this point as mentioned earlier, needs the confirmation of further research.

A fairly indiscriminate implication or rather suggestion of the present research is for teachers to allow learners do TE themselves. In other words, this is a kind of emphasizing important pints on learners' part mingled with TE on teacher or material developers' part. In simpler terms, the procedure is that teachers give learners a neutral text in which nothing is enhanced, then, they introduce the target structure to be learned and ask the learners to enhance the text wherever they find this structure. The disadvantage is that learners might grow more conscious of the form, and this is not what TE is trying to achieve. However, in this way each learner applies his/her own TE format, a format which is congruent with his/her learning strategy. This suggestion needs much research to be confirmed and paves the way for more studies that can delve beneath the relationship between learners' learning strategies and TE formats.

Nonetheless, the current study suffers from some limitations. One limitation of the study is the type of assessment used. As Leow (2001) commented, a more appropriate type of assessment would be to collect oral protocol by using on line measurement instruments since post exposure measurement instruments do not provide information about on line input processing and what learners pay attention to. Therefore, one way for researchers to find out what input learners pay attention to and notice is to collect oral protocol reports while the learners are doing the task (Jourdenaise, 2001). Another limitation of this study is that we used an immediate post test design which behooves future researchers to assess differential effects of these two new TE formats using delayed posttest designs. A third limitation is the fact that the participants were all female.

A TE format deserving further research is choice TE format which had very little effect on triggering the noticing of the target feature while it introduces both correct and incorrect forms and is almost semi-explicit. In other words, this TE format at first look seems to be able to trigger noticing more than other TE formats since it is more explicit than other TE formats.

\section{REFERENCES}


[1] Alanen, R. (1995). Input enhancement and rule presentation in second language acquisition. In R. Schmidt (Ed.), Attention and awareness in foreign language acquisition (pp. 259-302). University of Hawaii, Honolulu.

[2] Doughty, C. (1991). Second language instruction does make a difference: Evidence from an empirical study of SL relativization. Studies in Second Language Acquisition 13, 431-496.

[3] Ellis, R. (1997). SLA research and language teaching. Oxford: Oxford University Press.

[4] Foster, J. \& Coles, P. (1977). An experimental studying of typographical cueing in printed materials. Ergonomics 20, 57-66.

[5] Fotos, S. (1994). Integrating grammar instruction and communicative language use through grammar consciousness raising tasks. TESOL Quarterly, 28(2), 323-351.

[6] Fotos, S. (1998). Shifting the focus from forms to form in the EFL classrooms. ELT Journal, 52, 301-307.

[7] Han, Z. Park, E. S. \& Combs, C. H. (2008). Textual Enhancement of Input: Issues and Possibilities. Applied Linguistics, 29(4), 597-618.

[8] Izumi, S. (2002). Output, input enhancement, and the noticing hypothesis: An experimental study on ESL relativization. Studies in Second Language Acquisition, 24(4), 541-77.

[9] Izumi, S. (2003). Visual input enhancement as focus on form. Sophia Linguistica, 51, 1-30.

[10] Jourdenais, R. (1998). The effects of textual enhancement on the acquisition of the Spanish preterit and imperfect. Unpublished doctoral dissertation, Georgetown University, Washington DC.

[11] Jourdenais, R. (2001). Cognition, instruction and protocol analysis. In P. Robinson (Ed.), Cognition and second language instruction (pp. 354-375). Cambridge: Cambridge University Press.

[12] Jourdenais, R. O. M. Stauffer, S. Boyson, B. \& Doughty, C. (1995). Does textual enhancement promote noticing? A thinkaloud protocol analysis. In R. Schmidt (Ed.), Attention and awareness in second language learning (Technical Report 9) (pp. 183-216). University of Hawaii, Second Language Teaching and Curriculum Center, Honolulu.

[13] Lee, S. K. (2007). Effects of textual enhancement and topic familiarity on Korean EFL students' reading comprehension and learning of passive voice. Language Learning, 57, 87-118.

[14] Lee, S. K., \& Huang, H. T. (2008). Visual input enhancement and grammar learning: A meta-analytic review. Studies in Second Language Acquisition, 30, 307-331.

[15] Leow, R. (1997a). The effects of input enhancement and text length on adult L2 readers' comprehension and intake in second language acquisition. Applied Language Learning, 8, 151-182.

[16] Leow, R. (1997b). Attention, awareness and foreign language behavior. Language Learning 47, 465-505.

[17] Leow, R. (2001). Do learners notice enhanced forms while interacting with the L2? An on-line and off-line study of the role of written input enhancement in L2 reading. Hispania, 84, 496-509.

[18] Leow, R. E. T. Nuevo, A. M. \& Tsai, Y. C. (2003). The roles of textual enhancement and type of linguistic item in adult L2 learners' comprehension and intake. Applied Language Learning, 13, 1-16.

[19] Lightbown, P., Spada, N. (1990). Focus on form and corrective feedback in communicative language teaching: Effects on second language learning. Studies in Second Language Acquisition, 12, 429-448.

[20] Nassaji, H. (1999). Toward integrating form focused instruction and communicative interaction in the second language classroom: Some pedagogical possibilities. The Canadian Moderrn Language Review, 55, 385-402.

[21] Overstreet, M. (1998). Text enhancement and content familiarity: The focus of learner attention. Spanish Applied Linguistics, 2 , 229-258.

[22] Pica, T. (2000). Tradition and transition in English language teaching methodology. System, 28, 1-18.

[23] Qi, D.S., Lapkin, Sh. (2001). Exploring the role of noticing in a three stage second language writing task. Journal of Second Language Writing, 10, 277-303.

[24] Robinson, P. (1996). Learning simple and complex second language rules under implicit, incidental, rule search, and instructed conditions. Studies in Second Language Acquisition 18, 27-67.

[25] Rosa, E., Oneil, M. (1999). Explicitness, intake and the issue of awareness: another piece to the puzzle. Studies in Second Language Acquisition 21, 511-556.

[26] Savignon, S. (1991). Communicative language teaching: State of the art. TESOL Quarterly, 25, 261-277.

[27] Schmidt, R. (1994a). Deconstructing consciousness in search of useful definitions for applied linguistics. AILA Review 11, 1126.

[28] Schmidt, R., (1994b). Implicit learning and the cognitive unconscious. In: Ellis, N. (Ed.), Implicit and Explicit Learning of Languages. Academic Press, London, pp. 165-209.

[29] Scmidt, R. (ED). (1995). Consciousness and Foreign Language Learning. A Tutorial on the Role of Attention and Awareness in Learning. In Attention and Awareness in Foreign Language Learning. University of Hawaii, Honolulu, pp. 1-63.

[30] Schmidt, R. (2001). Attention. In: Robinson, P. (Ed.), Cognition and Second Language Instruction. Cambridge University Press, Cambridge, pp.3-32.

[31] Schmidt, R., Frota, S (1986). Developing basic conversational ability in a second language: a case study of an adult learner of Portuguese. In: Day, R. (Ed.), Talking to Learn: Conversation in Second Language Acquisition. Newbury House, Rowley, MA.

[32] Shebilske, W.L., Rotondo,J.A. (1981). Typographical and spatial cues that facilitate learning from textbooks. Visible Language XV 41-54, 1-63.

[33] Simard, D. (2009). Differential effects of textual enhancement formats on intake. System, 37, 124-135.

[34] Sharwood Smith, M. (1981). Consciousness raising and the second language learner. Applied Linguistics 2, 159-168.

[35] Sharwood Smith, M. (1991). Speaking to many minds: On the relevance of different types of language information for the L2 learner. Second Language Research 7, 118-132.

[36] Sharwood Smith, M. (1993). Input enhancement in instructed SLA: Theoretical bases. Studies in Second Language Acquisition 15, 165-179.

[37] Shook, D. J. (1994). FL/L2 reading, grammatical information, and the input to intake phenomenon. Applied Language Learning, 5, 57-93. 
[38] White, J. (1996). An input enhancement study with ESL children: Effects on the acquisition of possessive determiners. Unpublished Doctoral Dissertation, McGill University, Montre'al, Que bec, Canada.

[39] White, J. (1998). Getting the learners' attention: A typographical input enhancement study. In C. Doughty \& J. Williams (Eds.), Focus-on-form in second language classroom acquisition (pp. 91-128). Cambridge: Cambridge University Press.

[40] Wong, W. (2000). The effects of textual enhancement and simplified input on L2 comprehension and acquisition of nonmeaningful grammatical form. Unpublished dissertation, University of Illinois, Urbana-Champaign, IL.

[41] Wong, W. (2003). Textual enhancement and simplified input: effects on L2 comprehension and acquisition of non-meaningful grammatical form. Applied Language Learning, 13, 17-45.

Aliakbar Khomeijani Farahani has a $\mathrm{PhD}$ and is a professor of applied linguistics at the University of Tehran. Ha has been a faculty member for 22 years and has published papers nationally and internationally. His research interests are teaching methodology, first language acquisition and second language acquisition.

Mehdi Sarkhosh is a PhD student in applied linguistics at the University of Tehran. He has been teaching English courses for 4 years in different universities in Tehran. His research interests are: second language acquisition, language methodology and vocabulary acquisition. 